\title{
Early oseltamivir treatment improves survival in critically ill patients with influenza pneumonia
}

Gerard Moreno ${ }^{1,15}$, Alejandro Rodríguez ${ }^{1,15}$, Jordi Sole-Violán², Ignacio Martín-Loeches $\mathbb{1}^{3}$, Emili Díaz ${ }^{4}$, María Bodí ${ }^{1}$, Luis F. Reyes $\mathbb{1}^{5}$, Josep Gómez ${ }^{1}$, Juan Guardiola ${ }^{6}$, Sandra Trefler ${ }^{1}$, Loreto Vidaur ${ }^{7}$, Elisabet Papiol ${ }^{8}$, Lorenzo Socias ${ }^{9}$, Carolina García-Vidal ${ }^{10}$, Eudald Correig ${ }^{1}$, Judith Marín-Corral ${ }^{11}$, Marcos I. Restrepo ${ }^{12}$, Jonathan S. Nguyen-Van-Tam ${ }^{13}$ and Antoni Torres ${ }^{14}$ on behalf of the GETGAG Working Group ${ }^{16}$

\section{ABSTRACT}

Background: The relationship between early oseltamivir treatment (within $48 \mathrm{~h}$ of symptom onset) and mortality in patients admitted to intensive care units (ICUs) with severe influenza is disputed. This study aimed to investigate the association between early oseltamivir treatment and ICU mortality in critically ill patients with influenza pneumonia.

Methods: This was an observational study of patients with influenza pneumonia admitted to 184 ICUs in Spain during 2009-2018. The primary outcome was to evaluate the association between early oseltamivir treatment and ICU mortality compared with later treatment. Secondary outcomes were to compare the duration of mechanical ventilation and ICU length of stay between the early and later oseltamivir treatment groups. To reduce biases related to observational studies, propensity score matching and a competing risk analysis were performed.

Results: During the study period, 2124 patients met the inclusion criteria. All patients had influenza pneumonia and received oseltamivir before ICU admission. Of these, 529 (24.9\%) received early oseltamivir treatment. In the multivariate analysis, early treatment was associated with reduced ICU mortality (OR $0.69,95 \%$ CI $0.51-0.95$ ). After propensity score matching, early oseltamivir treatment was associated with improved survival rates in the Cox regression (hazard ratio 0.77, 95\% CI 0.61-0.99) and competing risk (subdistribution hazard ratio $0.67,95 \%$ CI $0.53-0.85$ ) analyses. The ICU length of stay and duration of mechanical ventilation were shorter in patients receiving early treatment.

Conclusions: Early oseltamivir treatment is associated with improved survival rates in critically ill patients with influenza pneumonia, and may decrease ICU length of stay and mechanical ventilation duration.

@ERSpublications

Controversy persists regarding the effectiveness of NAIs in the treatment of severe influenza due to a lack of data from RCTs. This propensity score-matched observational study reaffirms that early oseltamivir treatment is associated with better outcomes. https://bit.ly/2KdW5AJ

Cite this article as: Moreno G, Rodríguez A, Sole-Violán J, et al. Early oseltamivir treatment improves survival in critically ill patients with influenza pneumonia. ERJ Open Res 2021; 7: 008882020 [https://doi.org/10.1183/23120541.00888-2020].

This article has supplementary material available from openres.ersjournals.com

Received: 27 Nov 2020 | Accepted after revision: 7 Dec 2020

Copyright $\odot$ ERS 2021. This article is open access and distributed under the terms of the Creative Commons Attribution Non-Commercial Licence 4.0. 


\section{Introduction}

Influenza is one of the leading causes of mortality from infectious diseases worldwide, resulting in an estimated 300000-645000 deaths in adults each year [1]. Up to a third of adults hospitalised with influenza require admission to an intensive care unit (ICU), of whom $>60 \%$ require mechanical ventilation, frequently due to viral pneumonia and acute respiratory distress syndrome (ARDS) [2], with a resulting mortality rate of up to $30 \%$ [3]. Beyond supportive therapy, neuraminidase inhibitors (NAIs) are the first-line option for severe influenza [4-6]. Both the US Centers for Disease Control and Prevention and the World Health Organization recommend oseltamivir for patients hospitalised with severe, progressive, complicated influenza or for those at risk of developing complications. However, the timing of antiviral treatment may be critical in improving outcomes, as reports indicate that starting antivirals within $48 \mathrm{~h}$ of symptom onset can reduce mortality [7]. Thus, the mainstay of therapy for patients with severe influenza is the initiation of antiviral treatment as soon as possible after the onset of illness [8].

In the absence of strong evidence from randomised controlled trials (RCTs) involving critically ill patients, clinical knowledge relies on extrapolation from observational studies, which currently suggest that the effectiveness of NAIs is greatest when they are started within $48 \mathrm{~h}$ of symptom onset [3, 9-11]. A large meta-analysis, which included 5103 critically ill adults with influenza $\mathrm{A}(\mathrm{H} 1 \mathrm{~N} 1)$ pdm09, found a reduction in mortality of $38 \%$ in those receiving early NAI compared with those receiving treatment later [12]. However, this study has been widely criticised due to the high risk of biases [13, 14].

There has been a long-standing debate regarding the role and benefit of NAIs in the treatment of influenza. Current evidence suggests that the effect of NAIs on mortality depends on the population being treated and the clinical setting [15]. However, ethical constraints limit the ability to randomise severely ill patients and obtain the highest level of evidence from RCTs in intensive care settings. Therefore, to strengthen the evidence base, new observational studies need to focus on reducing biases and improving the quality of observational data.

In this study, we evaluated the association between early oseltamivir treatment and ICU mortality compared with later treatment in critically ill patients with influenza pneumonia. Our approach aimed to account for the biases present in previous observational studies.

\section{Materials and methods \\ Study design}

This was a retrospective analysis of prospectively collected data from subjects admitted to 184 ICUs in Spain ( $>50 \%$ of the country's ICUs) between June 2009 and April 2018. Data were obtained from a nationwide registry created by the Spanish Society of Intensive and Critical Care Medicine and Coronary Units (SEMICYUC). The information was recorded either by the attending physician or obtained by reviewing clinical case notes, laboratory data and radiological records. All consecutive cases admitted to an ICU who met the entry criteria were enrolled. The study was approved by the Ethics Committee of Joan XXIII University Hospital (Tarragona, Spain) (approval 11809). All data were anonymised, allowing the requirement for informed consent to be waived. The ICU admission criteria and treatment decisions were not standardised between the centres, and were left to the discretion of the attending physicians in accordance with the recommendations of the SEMICYUC [16].

\footnotetext{
Affiliations: ${ }^{1}$ Critical Care Dept, Hospital Universitari de Tarragona Joan XXIII, URV/IISPV/CIBERES, Tarragona, Spain. ${ }^{2}$ Critical Care Dept, Hospital Universitario de Gran Canaria Dr Negrín, CIBERES, Las Palmas de Gran Canaria, Spain. ${ }^{3}$ Dept of Anaesthesia and Critical Care, St James's University Hospital, Trinity Centre for Health Sciences, Multidisciplinary Intensive Care Research Organisation (MICRO), Dublin, Ireland. ${ }^{4}$ Critical Care Dept, Hospital Parc Taulí, CIBERES, Sabadell, Spain. ${ }^{5}$ Microbiology Dept, Universidad de La Sabana, Bogotá, Colombia. 'Division of Pulmonary, Critical Care and Sleep Medicine, University of Louisville and Robley Rex VA Medical Center, Louisville, KY, USA. ${ }^{7}$ Critical Care Dept, Hospital Universitario Donostia, San Sebastián, Spain. ${ }^{8}$ Critical Care Dept, Hospital Vall d'Hebrón, Barcelona, Spain. ${ }^{9}$ Critical Care Dept, Hospital Son Llàtzer, Palma de Mallorca, Spain. ${ }^{10}$ Infectious Diseases, Hospital Clínic de Barcelona, Barcelona, Spain. ${ }^{11}$ Critical Care Dept, Hospital Del Mar, Research Group in Critical Disorders (GREPAC), IMIM, Barcelona, Spain. ${ }^{12}$ South Texas Veterans Health Care System, University of Texas Health Sciences at San Antonio, San Antonio, TX, USA. ${ }^{13}$ Health Protection and Influenza Research Group, Epidemiology and Public Health, School of Medicine, University of Nottingham, Nottingham, UK. ${ }^{14}$ Servei de Pneumologia i Al-lèrgia Respiratòria, Institut Clínic del Tórax, Hospital Clínic de Barcelona, CIBERES, Barcelona, Spain.

${ }^{15}$ These authors contributed equally. ${ }^{16}$ GETGAG Study Group Investigators are listed in the supplementary material.
}

Correspondence: Gerard Moreno, Critical Care Dept, Hospital Universitari de Tarragona Joan XXIII, URV/ IISPV/CIBERES, C/ Dr Mallafrè Guasch 4, Tarragona 43005, Spain. E-mail: murenu77ahotmail.com 


\section{Subjects}

The inclusion criteria were: patients admitted to an ICU with a severe acute respiratory infection [17], acute respiratory failure, radiological findings consistent with pneumonia and microbiological confirmation of influenza A or B by reverse transcription (RT)-PCR at ICU admission, and receipt of oseltamivir prior to ICU admission. Nasopharyngeal swab specimens were collected at admission and lower respiratory tract secretions were also obtained in intubated patients. The exclusion criteria were: children $<15$ years old, patients with missing data (about antiviral therapy or the start of antiviral therapy, time from symptom onset to hospital and ICU admission, or incomplete follow-up), patients without pneumonia and those with an ICU length of stay $<48 \mathrm{~h}$.

\section{Data collection}

The following information was collected at and during ICU admission (supplementary table S1): demographic data, comorbidities, time from illness onset to hospital admission, time to first dose of antiviral delivery and dosage of oseltamivir, time from hospital admission to ICU admission, complications during the ICU stay, and microbiological, laboratory and chest radiological findings. Illness severity was determined with the Acute Physiology and Chronic Health Evaluation (APACHE) II score, which was calculated for all patients within the first $24 \mathrm{~h}$ of ICU admission. Organ failure was assessed using the Sequential Organ Failure Assessment (SOFA) score.

\section{Study definitions}

Two treatment groups were defined: 1) the early oseltamivir group (who received oseltamivir within 2 days of symptom onset) and 2) the later oseltamivir group (who received oseltamivir after 2 days since symptom onset).

Primary viral pneumonia was defined as acute respiratory failure and the presence of pulmonary infiltrates on chest radiography in combination with a positive RT-PCR test result for influenza and negative respiratory and blood bacterial cultures during the acute phase of infection [18]. Community-acquired respiratory co-infection (CARC) or bacterial co-infection was defined as any infection diagnosed within the first 2 days of hospitalisation by isolation of respiratory or blood cultures positive for bacteria/fungi during ICU admission [19]. All remaining definitions are summarised in the supplementary material.

\section{Outcomes}

The primary outcome was to investigate the association between early oseltamivir treatment and ICU mortality compared with later treatment. Secondary outcomes were to analyse: 1) factors associated with later oseltamivir administration, 2) ICU length of stay in the two treatment groups and 3) duration of mechanical ventilation in survivors in the two treatment groups.

\section{Statistical analysis}

Discrete variables are expressed as count (percentage), while continuous variables are presented as median (interquartile range (IQR)). For the demographic and clinical characteristics, differences between the groups were assessed with the Chi-squared test and Fisher's exact test for categorical variables or the t-test or Mann-Whitney U-test for continuous variables. Time zero of follow-up was ICU admission. To avoid immortal time bias, we controlled for internal left-truncation in the data from patients who initiated oseltamivir treatment after this time-point. Thus, all patients have initiated oseltamivir at time zero of the follow-up. Data from patients with ICU length of stay $<48 \mathrm{~h}$ were also left-truncated to ensure that the effect of treatment exposure was guaranteed. A binary logistic regression was used to analyse the factors associated with later oseltamivir initiation. In the unmatched cohort, a multiple logistic regression analysis was performed to evaluate the association between early oseltamivir treatment and ICU mortality. The results of these analyses are presented as the odds ratio with $95 \%$ confidence interval. Model integrity was tested using standard diagnostic statistics, and plots and the goodness of fit for each model were assessed for all outcomes with the Hosmer-Lemeshow test.

Propensity score matching was used to address confounding by indication when investigating treatment effects $[20,21]$. A $2: 1$ matched analysis without replacement and with a caliper of 0.2 was performed using an optimal matching algorithm between patients in the early and later oseltamivir treatment groups using estimated propensity scores. To predict the likelihood of treatment, we performed a multivariate logistic regression analysis regularised by the lasso method that included all the covariates. For the matched set, we used two types of survival models: a Cox proportional hazards model applied to a cause-specific hazard (CSH) function and a competing risk analysis using a Fine-Gray model [22-24]. Models were constructed for a 60-day follow-up. The CSH model considered ICU discharge as a censoring event, allowing the interpretation of aetiological relationships between early oseltamivir use and patient outcomes. The results are presented as the hazard ratio (HR) with 95\% confidence interval. The competing 
risk analysis was performed by treating ICU mortality and discharge as competing events [13, 25]. The Fine-Gray model calculates the probability of any event (death or discharge) at time $t$ based on the assumption that no other event has occurred and, therefore, it can be used to predict events. This procedure was performed using a subdistribution hazard function (subdistribution HR (sHR)). Cumulative incidences were plotted for early versus later oseltamivir treatment and for both competing events. We checked the proportional hazards assumption model and the proportionality of the subdistribution from the Fine-Gray model. No violations were found.

Data analysis was performed with SPSS version 24 (IBM, Armonk, NY, USA), except for the survival models, for which $\mathrm{R}$ version 3.6 (cran.r-project.org) was used.

\section{Results}

During the study period, 4175 patients were admitted to an ICU for severe influenza. Among them, 2124 met the inclusion criteria of this study (figure 1).

\section{Baseline characteristics}

The median (IQR) age of the cohort was 54 (43-65) years and 1299 (61.2\%) were male. The most frequent underlying chronic disease was obesity $(\mathrm{n}=675(31.8 \%))$, followed by chronic obstructive pulmonary disease (COPD) ( $\mathrm{n}=393$ (18.5\%)). The median (IQR) APACHE II and SOFA scores were 16 (11-21) and 6 (4-8), respectively. Regarding the influenza strain, influenza $\mathrm{A}(\mathrm{H} 1 \mathrm{N1}) \mathrm{pdm} 09$ was the most frequently isolated $(\mathrm{n}=1533(72.2 \%))$, followed by influenza $\mathrm{A}(\mathrm{H} 3 \mathrm{~N} 2)(\mathrm{n}=336(15.8 \%))$ and influenza $\mathrm{B}(\mathrm{n}=148$ (7\%)). The remaining strains $(\mathrm{n}=107(5 \%))$ were nontypeable influenza A. Only 308 (14.5\%) patients in the full cohort had been vaccinated against influenza.

All patients were diagnosed with influenza-related pneumonia, of whom 1586 (74.7\%) had primary viral pneumonia and 538 (25.3\%) had CARC. The median (IQR) number of lobes showing pulmonary infiltrates on chest radiographs was $2(2-4)$. The rate of invasive mechanical ventilation was $59.1 \%$, with 618 (29.1\%) patients receiving prone therapy. At ICU admission, septic shock was frequent $(\mathrm{n}=1157$ (54.5\%)) and 611 (28.7\%) patients developed acute renal failure. All patients received oseltamivir before ICU admission (supplementary figure S1) and early oseltamivir treatment was administered in 529 (24.9\%) patients.

\section{Factors associated with later oseltamivir initiation}

Before the propensity score matching, a multivariate logistic regression analysis was performed in the unmatched cohort to determine the factors associated with later antiviral therapy. The baseline

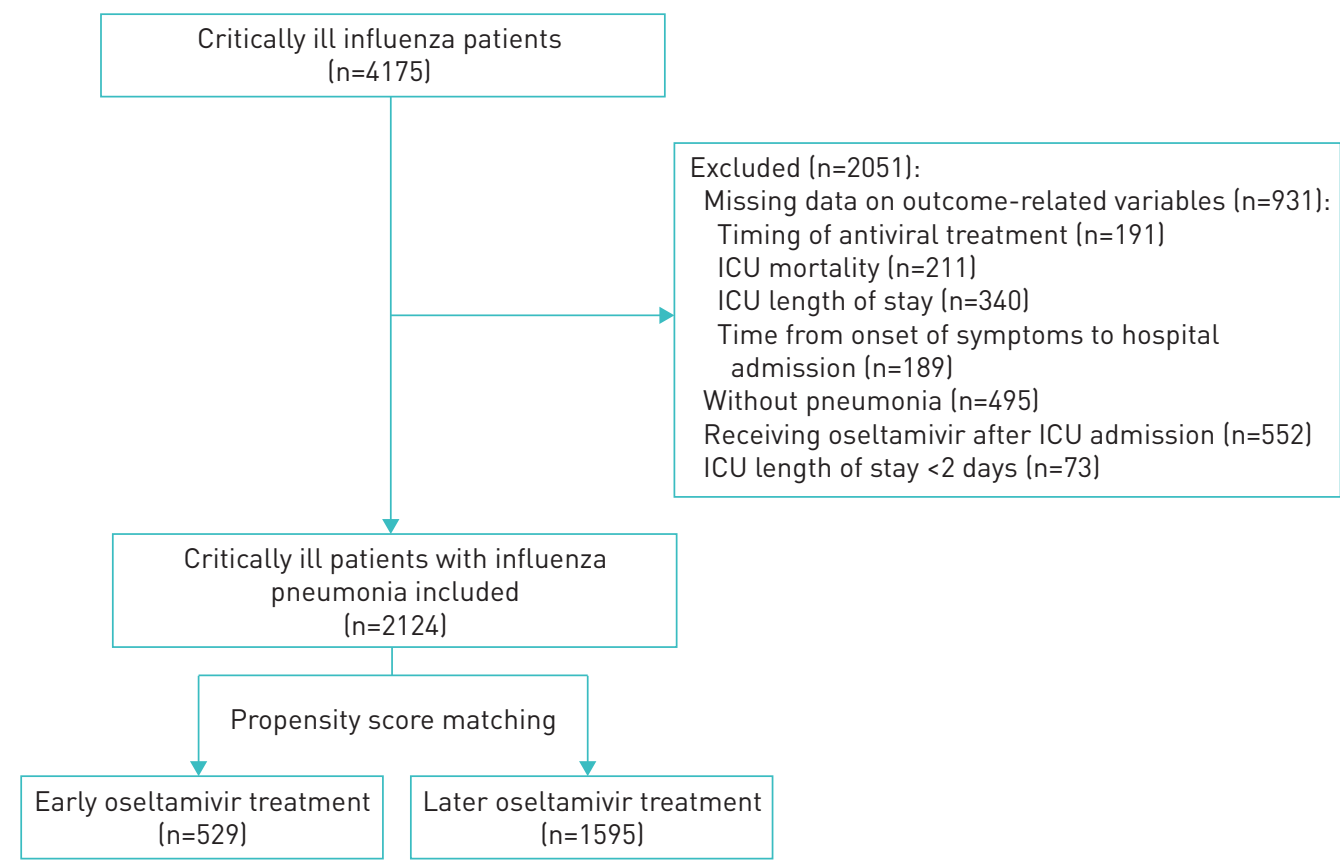

FIGURE 1 Study flowchart. ICU: intensive care unit. The early treatment group comprised patients who received oseltamivir within the first 2 days of illness onset. The later treatment group comprised patients who received oseltamivir after 2 days since illness onset. 
demographic factors included in the model were age, immunosuppression, asthma, COPD, congestive heart failure and obesity. The only variable associated with later oseltamivir treatment was obesity (OR 1.49, 95\% CI 1.18-1.87; p=0.01) (supplementary table S2).

\section{Mortality analysis}

The overall ICU mortality rate was $21.3 \%$. Despite the absence of significant differences, there was a trend towards a lower mortality rate with early oseltamivir treatment compared with later treatment in the subgroups based of age and severity of illness (except for those extremely ill with an APACHE II score $>30$ ), as shown in supplementary figures S2 and S3. In the unmatched cohort, there were significant differences between survivors and nonsurvivors (supplementary table S3). The multivariate logistic regression analysis was adjusted for age, sex, APACHE II and SOFA scores, chronic kidney disease, immunosuppression, pregnancy, number of pulmonary infiltrates, mechanical ventilation, acute renal failure, septic shock, CARC, ventilator-associated pneumonia, oseltamivir treatment duration, and adjuvant corticosteroid treatment. It revealed a significant association between early oseltamivir treatment and lower ICU mortality compared with later treatment (OR 0.69, 95\% CI 0.51-0.95; $\mathrm{p}=0.02$ ) (figure 2).

Propensity score matching was performed by matching 529 patients in the early oseltamivir treatment group with 1058 patients in the later oseltamivir treatment group. Table 1 shows the baseline characteristics and complications at ICU admission of the unmatched and matched cohorts. The study groups were well balanced in terms of demographic characteristics, severity of illness, comorbidities and complications at ICU admission (table 1 and supplementary figure S4). The median time from hospital to ICU admission was 1 day in both groups. However, this median time showed a statistically significant difference between the groups, being longer in the early oseltamivir treatment group. The ICU mortality rate was significantly different between the early and later oseltamivir treatment groups in the propensity score-matched cohort $(19.1 \%$ versus $24.6 \% ; \mathrm{p}=0.01)$. ICU length of stay and duration of mechanical ventilation were both significantly reduced by early oseltamivir treatment compared with later treatment (median (IQR) 8 (4-16) versus 10 (5-21) days and 5.7 (2.7-12) versus 8 (3-18) days, respectively).

The effect of early oseltamivir treatment on overall mortality was evaluated using the CSH model. There was a significant difference in the 60-day mortality rate between the groups. A forest plot showing the effects of different clinical variables included in the model (supplementary figure S5) demonstrated the

Early oseltamivir treatment

Chronic kidney disease

Oseltamivir treatment duration

$$
\begin{array}{r}
\text { Male } \\
\text { Age } \\
\text { Bacterial co-infection } \\
\text { SOFA score } \\
\text { APACHE II score }
\end{array}
$$$$
\text { Age }
$$

Number of pulmonary infiltrates

Corticosteroid treatment

Ventilator-associated pneumonia

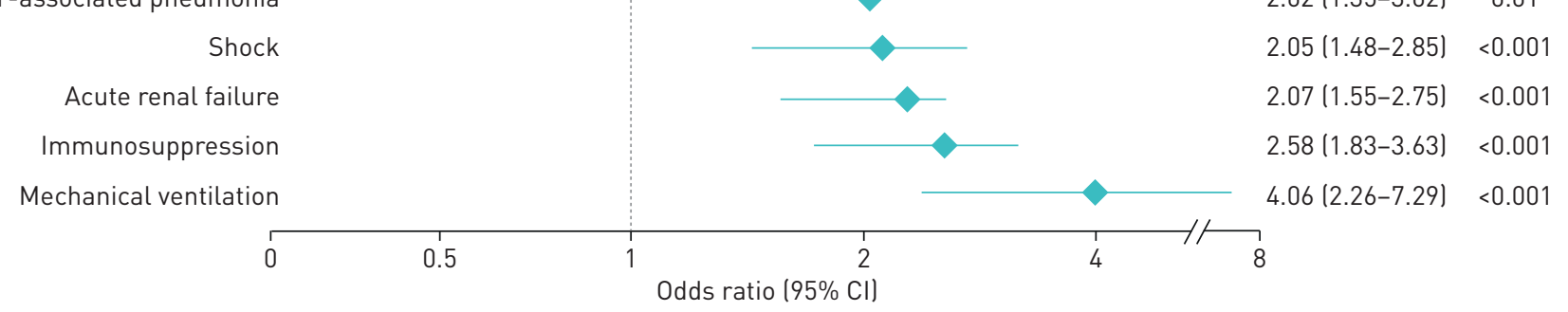

FIGURE 2 Multivariate logistic regression analysis for factors associated with intensive care unit mortality in the unmatched cohort. SOFA: Sequential Organ Failure Assessment; APACHE: Acute Physiology and Chronic Health Evaluation. 
TABLE 1 Baseline characteristics of the unmatched and propensity score-matched groups of patients ${ }^{\#}$

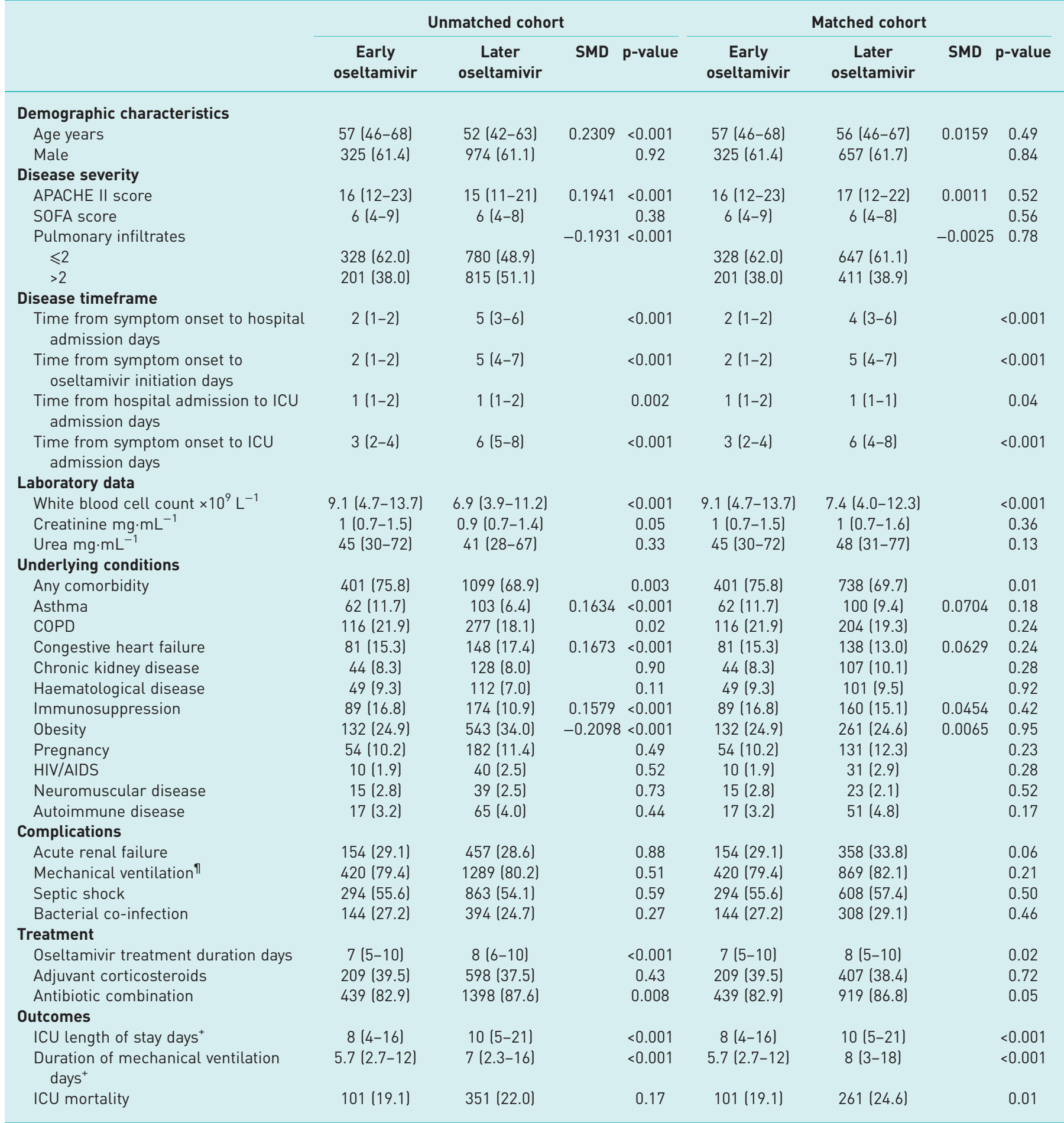

Data are presented as median (interquartile range) or $\mathrm{n}(\%)$, unless otherwise stated. SMD: standardised mean difference; APACHE: Acute Physiology and Chronic Health Evaluation; SOFA: Sequential Organ Failure Assessment; COPD: chronic obstructive pulmonary disease; ICU: intensive care unit. * : unmatched cohort $n=2124$ (early oseltamivir $n=529$; later oseltamivir $n=1595$ ) and matched cohort $n=1587$ (early oseltamivir $n=529$; later oseltamivir $n=1058$ ); " : noninvasive and invasive mechanical ventilation; ${ }^{+}$: only in survivors.

significant protective effect of early oseltamivir treatment (HR 0.77, 95\% CI 0.61-0.99; p=0.03). A CSH model for ICU discharge also showed beneficial effects of early oseltamivir treatment (HR 1.21, 95\% CI 1.07-1.36; $\mathrm{p}=0.002$ ) (supplementary figure S6). Additionally, we performed a competing risk analysis to compare the probability of event with the probability of being discharged from hospital (figure 3 and 


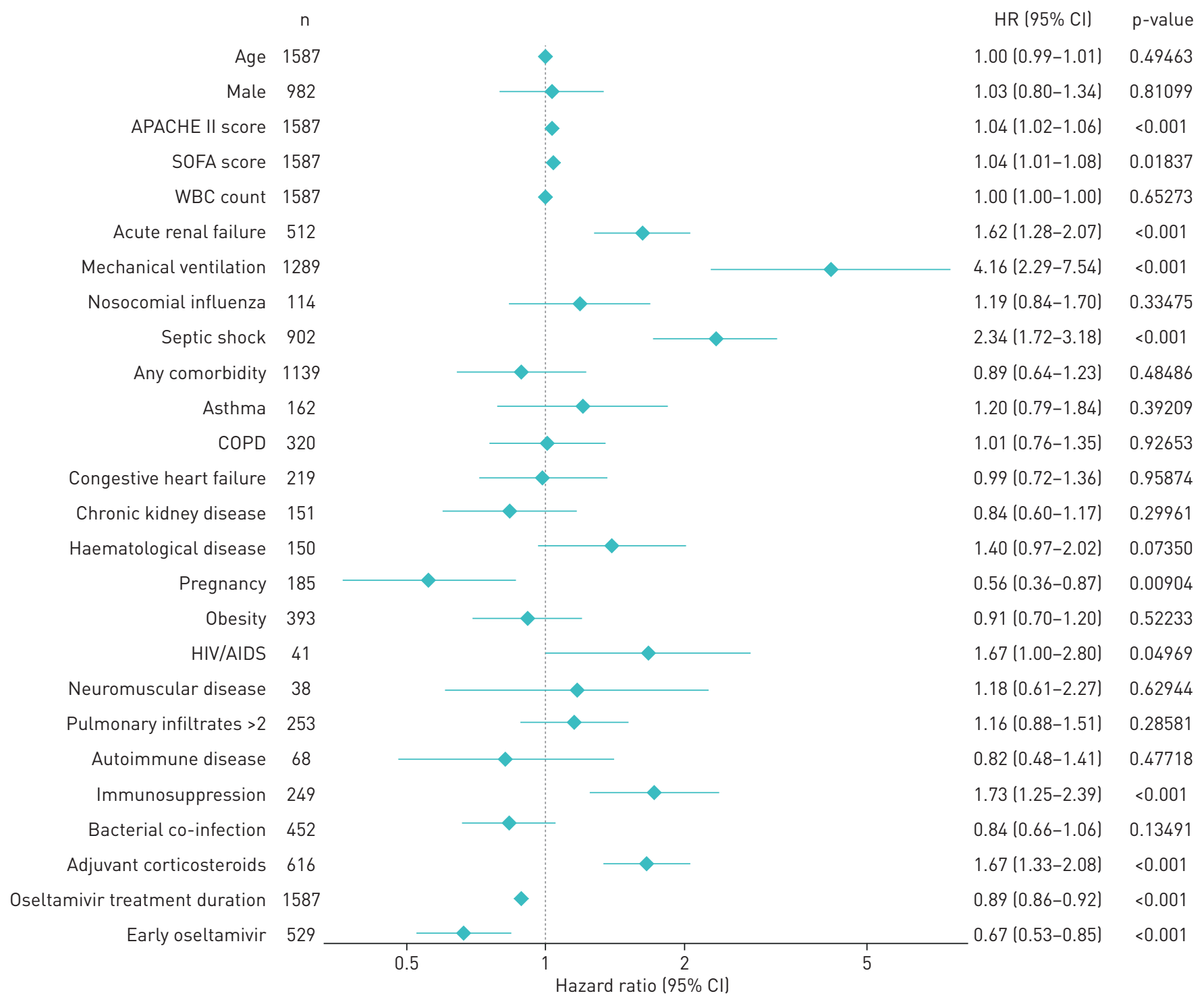

FIGURE 3 Forest plot of the competing risks analysis for the association of early oseltamivir treatment and 60-day intensive care unit mortality. APACHE: Acute Physiology and Chronic Health Evaluation; SOFA: Sequential Organ Failure Assessment; WBC: white blood cell; COPD: chronic obstructive pulmonary disease.

supplementary figure S7). Models were adjusted for age, sex, APACHE II and SOFA scores, white blood cell count, acute renal failure, mechanical ventilation, nosocomial influenza, septic shock, presence of any comorbidity, asthma, COPD, congestive heart failure, chronic kidney disease, haematological disease, pregnancy, obesity, HIV/AIDS, neuromuscular disease, pulmonary infiltrates (more than two lobes involved), autoimmune disease, immunosuppression, bacterial co-infection, adjuvant corticosteroids, and oseltamivir treatment duration. The predicted survival curves showed that early oseltamivir treatment was associated with a significantly lower probability of ICU mortality ( $\mathrm{sHR} 0.67,95 \%$ CI $0.53-0.85 ; \mathrm{p}<0.001$ ) and a higher probability of ICU discharge (sHR 1.28, 95\% CI 1.13-1.44; p<0.001) compared with later treatment (figure 4).

\section{Discussion}

Early oseltamivir treatment in critically ill adults with influenza pneumonia was significantly associated with a reduction of $33 \%$ in ICU mortality compared with later treatment. This association was evident in a large, broadly representative sample of critically ill patients with pandemic and seasonal influenza after adjusting for disease severity and controlling for confounders and other time biases. Furthermore, the effect was consistently seen across a broad range of analytical approaches, adding substantial robustness to the overall findings. 

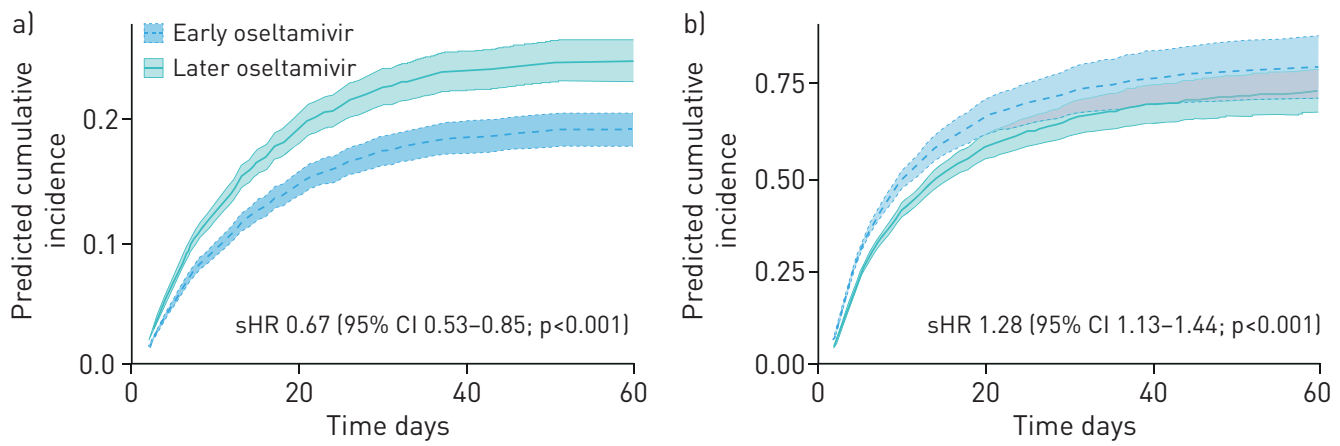

FIGURE 4 Survival plots of 60-day intensive care unit (ICU) mortality rates from the competing risk analysis between the early and later oseltamivir treatment groups: al predicted cumulative incidence of ICU mortality for both treatment groups and b) predicted cumulative incidence of being discharged alive from the ICU. sHR: subdistribution hazard ratio. sHRs are adjusted for age, sex. Acute Physiology and Chronic Health Evaluation II scores, Sequential Organ Failure Assessment scores, white blood cell count, acute renal failure, mechanical ventilation, nosocomial influenza, septic shock, presence of any comorbidity, asthma, chronic obstructive pulmonary disease, congestive heart failure, chronic kidney disease, haematological disease, pregnancy, obesity, HIV/AIDS, neuromuscular disease, pulmonary infiltrates (more than two lobes involved), autoimmune disease, immunosuppression, bacterial co-infection, adjuvant corticosteroids, and oseltamivir treatment duration.

Our findings are consistent with those of several observational studies on the effectiveness of NAIs published since the influenza $\mathrm{A}(\mathrm{H} 1 \mathrm{~N} 1)$ pdm09 pandemic $[3,9,26,27]$. LeE et al. [28] reported that NAI treatment (predominantly oseltamivir) was independently associated with survival in hospitalised patients (with a low proportion of critically ill patients), with the highest benefit observed when treatment was started within the first 2 days of illness onset (HR 0.20 , 95\% CI $0.12-0.32$ ). However, there was also benefit with treatment within 3-5 days compared with no treatment. This observation was made after carefully controlling for confounding factors and immortal time bias. MUTHuRI et al. [12] performed a large meta-analysis of individual participant data which included 5103 adults with influenza A(H1N1) pdm09 admitted to an ICU. They found a reduction in mortality of 38\% in those receiving early NAI compared with those receiving treatment later. Despite controlling for potential biases related to the timing of therapy and death, the study has been widely criticised due to the high risk of selection biases, possible confounding by indication, and possible immortal time bias [13, 14].

Most previous studies have focused only on influenza A(H1N1)pdm09. Recently, LYTRAs et al. [29] published data from 1330 adults in ICUs with severe influenza. They observed a $30 \%$ reduction in mortality with early oseltamivir treatment compared with later therapy among patients with influenza A $(\mathrm{H} 3 \mathrm{~N} 2)$, although this benefit was not found in patients with influenza B or $\mathrm{A}(\mathrm{H} 1 \mathrm{~N} 1)$, suggesting that the effectiveness of oseltamivir may vary between influenza viral strains. However, the authors did not adjust for baseline severity (e.g. APACHE II or SOFA scores), which is important for predicting mortality. In addition to using mechanically ventilated patients as a marker of severity, it is very important to adjust for severity given that patients can develop pneumonia, ARDS, septic shock and multiorgan failure [30]. In our study, after accounting for this severity and other confounding factors with propensity score matching, we observed a clear association between early oseltamivir therapy and lower ICU mortality for the different virus strains, predominantly influenza $\mathrm{A}(\mathrm{H} 1 \mathrm{~N} 1) \mathrm{pdm} 09$. It is noteworthy that the mortality rate in the study of LYTRAS et al. [29] was $46 \%$ compared with the ICU mortality rate of $21.3 \%$ in our study. Moreover, LYTRAS et al. [29] did not mention any possible complications during the ICU stay that could explain the high mortality rate. This might be the reason for the differences with our results regarding the usefulness of early oseltamivir treatment for the different types of influenza strains. Data from the FluSurv-NET indicated that influenza A and B resulted in similar morbidity and mortality rates among hospitalised patients; therefore, therapeutic intervention should not be influenced by the type of influenza virus [31].

Early oseltamivir treatment appeared to be beneficial in our study, based on multiple analytical approaches. This suggests that patients with severe influenza pneumonia should be treated immediately based on clinical suspicion during influenza epidemics, without waiting for a diagnostic confirmation. This is in line with current guidance and expert reviews that recommend starting treatment as soon as possible $[4,7,8,16]$. Even in the cases of delayed hospital admission, oseltamivir should be initiated promptly because viral shedding may be prolonged in severe or potentially fatal cases [32-34]. This was observed by MUTHURI et al. [12], who reported that mortality was reduced in ICU patients when treatment was commenced late (versus no treatment). Moreover, virological studies have found a strong correlation 
between viral replication, clinical symptoms and disease severity [35]. Despite these recommendations, the rate of early antiviral treatment remains low, ranging from $17 \%$ to $33.7 \%$ in previous reports $[9,12,29,36$, 37]. Indeed, when influenza is circulating in the community, it is suggested that early NAI treatment should be emphasised for outpatients who are at risk or present with severe disease, while hospitalised patients should be treated promptly without waiting for influenza test results $[38,39]$. Some studies have suggested that the delayed initiation of antiviral therapy may contribute to increased illness severity and worse clinical outcomes [36, 40]. Early NAI therapy has been associated with a substantially decreased length of hospital stay [37,41]. We also observed this in our study, as early oseltamivir treatment was associated with an increased probability of being discharged alive from the ICU and shorter durations of mechanical ventilation and ICU stays.

We noted that obesity was the only variable independently associated with the later initiation of oseltamivir treatment. Although critically ill obese patients might not have increased mortality, there may be an association between obesity and a higher consumption of ICU resources [42]. Thus, oseltamivir treatment should be immediately started based on a clinical suspicion of influenza in patients at risk of developing complications without waiting for virological confirmation, especially in obese patients who are at risk of receiving antiviral treatment later.

This is one of the largest observational studies of critically ill patients with both pandemic and seasonal influenza pneumonia. We evaluated the effectiveness of early oseltamivir treatment by carefully controlling for important confounders by propensity score matching, as well as for immortal time bias and competing risks. The study was also performed in multiple centres that provided data about influenza type and time from symptom onset to treatment. Therefore, this study improves on previous investigations by addressing the issues of those earlier studies.

However, we must acknowledge some limitations of our study. First, we did not review the duration of viral shedding or the appearance of drug-resistant viral strains in the groups. Fortunately, the incidence of oseltamivir resistance has remained low since the emergence of influenza A(H1N1)pdm09 [5]. Nevertheless, antiviral resistance should be an area for concern and must be considered in patients with immunosuppression or severe disease. Second, we did not collect data on oseltamivir side-effects or adjustment for patients with kidney failure and/or kidney replacement therapy. Oseltamivir is generally well tolerated, with the most common side-effects being gastrointestinal (e.g. nausea and vomiting). Evidence also suggests that drug clearance is significantly reduced in patients receiving kidney replacement therapy, necessitating dose adjustments. Third, the high number of excluded patients can result in attrition bias. However, there were no clinically relevant differences between the included and excluded patients in terms of age, illness severity and comorbidities. Fourth, despite effectively addressing several known potential biases, the nature of the observational study design meant that residual confounding and bias could have remained. However, the propensity score matching analysis should have balanced the population and observed confounders, and should have yielded the best evidence to date for physicians in the absence of firm data from RCTs.

In conclusion, among critically ill adults with influenza pneumonia, early oseltamivir therapy compared with later treatment was associated with improved survival rates and a considerable reduction in the use of healthcare resources, as evidenced by the reduced durations of both mechanical ventilation and ICU stay.

Acknowledgements: The authors thank Michael Maudsley and Tasneem Ahmed (University of Barcelona, Barcelona, Spain) for editing the manuscript in English, and the GETGAG working group.

Support statement: This study was supported, in part, by grants from the Spanish Society of Intensive and Critical Care Medicine and Coronary Units (SEMICYUC) and the Ricardo Barri Casanovas Foundation. The study sponsors had no role in the study design, data collection, analysis or interpretation, or the writing of the report. Funding information for this article has been deposited with the Crossref Funder Registry.

Author contributions: G. Moreno, A. Rodríguez, J. Sole-Violán, I. Martín-Loeches, E. Díaz and A. Torres conceived and designed the study. All authors, apart from M.I. Restrepo, L.F. Reyes, J. Gómez, C. García-Vidal, E. Correig and J.S. Nguyen-Van-Tam, contributed to the acquisition and local preparation of the constituent database. G. Moreno, A. Rodríguez, E. Correig, S. Trefler, I. Martín-Loeches, E. Díaz and J. Gómez contributed to the database creation and standardisation, the design of statistical analyses, and data analysis. G. Moreno, A. Rodríguez, L.F. Reyes, J.S Nguyen-Van-Tam, J. Sole-Violán, E. Díaz, M. Bodí, S. Trefler, J. Gómez, J. Guardiola, E. Correig, A. Torres, J. Marín-Corral, M.I. Restrepo, L. Vidaur, E. Papiol, L. Socias, C. García-Vidal and I. Martín-Loeches made important intellectual contributions, actively participated in the interpretation of the data and wrote the paper. All authors contributed to the critical review of the paper for important intellectual content and approved the final manuscript. The corresponding author (G. Moreno) had full access to all the study data and had the final responsibility for the decision to submit for publication.

Conflict of interest: G. Moreno has nothing to disclose. A. Rodríguez has nothing to disclose. J. Sole-Violán has nothing to disclose. I. Martín-Loeches has nothing to disclose. E. Díaz has nothing to disclose. M. Bodí has nothing to disclose. 
L.F. Reyes has nothing to disclose. J. Gómez has nothing to disclose. J. Guardiola has nothing to disclose. S. Trefler has nothing to disclose. L. Vidaur has nothing to disclose. E. Papiol has nothing to disclose. L. Socias has nothing to disclose. C. García-Vidal reports grants and other support from Gilead Science and Merck Sharp \& Dohme, and other support from Novartis, Pfizer, Janssen and Lilly, outside the submitted work. E. Correig has nothing to disclose J. Marín-Corral has nothing to disclose. M.I. Restrepo has nothing to disclose. J.S. Nguyen-Van-Tam reports grants from F. Hoffmann-La Roche, outside the submitted work. He was seconded to the Dept of Health and Social Care (DHSC) England in 2017; the views in this article are those of the authors and not necessarily those of the DHSC A. Torres has nothing to disclose.

\section{References}

1 Iuliano $\mathrm{AD}$, Roguski $\mathrm{KM}$, Chang $\mathrm{HH}$, et al. Estimates of global seasonal influenza-associated respiratory mortality: a modelling study. Lancet 2018; 391: 1285-1300.

2 Herold S, Becker C, Ridge KM, et al. Influenza virus-induced lung injury: pathogenesis and implications for treatment. Eur Respir J 2015; 45: 1463-1478.

3 Rodríguez A, Díaz E, Martín-Loeches I, et al. Impact of early oseltamivir treatment on outcome in critically ill patients with 2009 pandemic influenza A. J Antimicrob Chemother 2011; 66: 1140-1149.

4 Fry AM, Riley LE, McGeer A, et al. Clinical Practice Guidelines by the Infectious Diseases Society of America: 2018 Update on Diagnosis, Treatment, Chemoprophylaxis, and Institutional Outbreak Management of Seasonal Influenza. Clin Infect Dis 2018; 68: e1-e47.

5 Mc Mahon A, Martin-Loeches I. The pharmacological management of severe influenza infection "existing and emerging therapies". Expert Rev Clin Pharmacol 2017; 10: 81-95.

6 O'Sullivan S, Torres A, Rodriguez A, et al. Influenza management with new therapies. Curr Opin Pulm Med 2020; 26: $215-221$

7 US Centers for Disease Control and Prevention. Influenza antiviral medications: summary for clinicians. 2020 www.cdc.gov/flu/professionals/antivirals/summary-clinicians.htm Date last accessed: December 23, 2020.

8 Chow EJ, Doyle JD, Uyeki TM. Improvement in the survival rates of extracorporeal membrane oxygenation-supported respiratory failure patients: a multicenter retrospective study in Korean patients. Crit Care 2019; 23: 1-11.

9 Louie JK, Yang S, Acosta M, et al. Treatment with neuraminidase inhibitors for critically ill patients with influenza A (H1N1)pdm09. Clin Infect Dis 2012; 55: 1198-1204.

10 Kumar A. Early versus late oseltamivir treatment in severely ill patients with 2009 pandemic influenza A (H1N1): speed is life. J Antimicrob Chemother 2011; 66: 959-963.

11 Muthuri SG, Venkatesan S, Myles PR, et al. Impact of neuraminidase inhibitors on influenza A(H1N1) pdm09-related pneumonia: an individual participant data meta-analysis. Influenza Other Respir Viruses 2016; 10: 192-204.

12 Muthuri SG, Venkatesan S, Myles PR, et al. Effectiveness of neuraminidase inhibitors in reducing mortality in patients admitted to hospital with influenza A H1N1pdm09 virus infection: a meta-analysis of individual participant data. Lancet Respir Med 2014; 2: 395-404.

13 Resche-Rigon M, Azoulay E, Chevret S. Evaluating mortality in intensive care units: contribution of competing risks analyses. Crit Care 2005; 10: 1-6.

14 Wolkewitz M, Schumacher M. Survival biases lead to flawed conclusions in observational treatment studies of influenza patients. J Clin Epidemiol 2017; 84: 121-129.

15 Louie JK, Lampiris H. Treating influenza with neuraminidase inhibitors what is the evidence? JAMA Intern Med 2015; 175: 1899-1900.

16 Rodríguez A, Álvarez-Rocha L, Sirvent JM, et al. Recommendations of the Infectious Diseases Work Group (GTEI) of the Spanish Society of Intensive and Critical Care Medicine and Coronary Units (SEMICYUC) and the Infections in Critically Ill Patients Study Group (GEIPC) of the Spanish Society of Infectious Diseases and Clinical Microbiology (SEIMC) for the diagnosis and treatment of influenza A/H1N1 in seriously ill adults admitted to the Intensive Care Unit. Med Intensiva 2012; 36: 103-137.

17 Fitzner J, Qasmieh S, Mounts AW, et al. Revision of clinical case definitions: influenza-like illness and severe acute respiratory infection. Bull World Health Organ 2018; 96: 122-128.

18 Moreno G, Rodríguez A, Reyes LF, et al. Corticosteroid treatment in critically ill patients with severe influenza pneumonia: a propensity score matching study. Intensive Care Med 2018; 44: 1470-1482.

19 Martin-Loeches I, Schultz MJ, Vincent JL, et al. Increased incidence of co-infection in critically ill patients with influenza. Intensive Care Med 2017; 43: 48-58.

20 Austin PC. An introduction to propensity score methods for reducing the effects of confounding in observational studies. Multivariate Behav Res 2011; 46: 399-424.

21 Austin PC, Stuart EA. Optimal full matching for survival outcomes: a method that merits more widespread use. Stat Med 2015; 34: 3949-3967.

22 Fine JP, Gray RJ. A proportional hazards model for the subdistribution of a competing risk. J Am Stat Assoc 1999; 94: 496-509.

23 Lau B, Cole SR, Gange SJ. Competing risk regression models for epidemiologic data. Am J Epidemiol 2009; 170: 244-256.

24 Austin PC, Lee DS, Fine JP. Introduction to the analysis of survival data in the presence of competing risks. Circulation 2016; 133: 601-609.

25 Jones M, Fowler R. Immortal time bias in observational studies of time-to-event outcomes. J Crit Care 2016; 36 195-199.

26 Dominguez-Cherit G, Lapinsky SE, Macias AE, et al. Critically ill patients with 2009 influenza A(H1N1) in Mexico. JAMA 2009; 302: 1880-1887.

27 Hanshaoworakul W, Simmerman JM, Narueponjirakul U, et al. Severe human influenza infections in Thailand: oseltamivir treatment and risk factors for fatal outcome. PLoS One 2009; 4: 1-7.

28 Lee N, Leo YS, Cao B, et al. Neuraminidase inhibitors, superinfection and corticosteroids affect survival of influenza patients. Eur Respir J 2015; 45: 1642-1652. 
29 Lytras T, Mouratidou E, Andreopoulou A, et al. Effect of early oseltamivir treatment on mortality in critically ill patients with different types of influenza: a multiseason cohort study. Clin Infect Dis 2019; 69: 1896-1902.

30 Martin-Loeches I, Díaz E, Vidaur L, et al. Pandemic and post-pandemic influenza A (H1N1) infection in critically ill patients. Crit Care 2011; 15: 1-11.

$31 \mathrm{Su}$ S, Chaves SS, Perez A, et al. Comparing clinical characteristics between hospitalized adults with laboratory-confirmed influenza A and B virus infection. Clin Infect Dis 2014; 59: 252-255.

32 Khoury J, Szwarcwort M, Kra-oz Z, et al. Duration of viral shedding and factors associated with prolonged shedding among inpatients with influenza treated with oseltamivir: a prospective cohort study. Eur J Clin Microbiol Infect Dis 2018; 37: 319-323.

$33 \mathrm{Hu} \mathrm{Y,} \mathrm{Lu} \mathrm{S}$, Song Z, et al. Association between adverse clinical outcome in human disease caused by novel influenza A H7N9 virus and sustained viral shedding and emergence of antiviral resistance. Lancet 2013; 381: 2273-2279.

34 Ling LM, Chow AL, Lye DC, et al. Effects of early oseltamivir therapy on viral shedding in 2009 pandemic influenza A (H1N1) virus infection. Clin Infect Dis 2010; 50: 963-969.

35 Carrat F, Vergu E, Ferguson NM, et al. Time lines of infection and disease in human influenza: a review of volunteer challenge studies. Am J Epidemiol 2008; 167: 775-785.

36 Yu H, Feng Z, Uyeki TM, et al. Risk factors for severe illness with 2009 pandemic influenza A (H1N1) virus infection in China. Clin Infect Dis 2011; 52: 457-465.

37 Dou L, Reynolds D, Wallace L, et al. Decreased hospital length of stay with early administration of oseltamivir in patients hospitalized with influenza. Mayo Clin Proc Innov Qual Outcomes 2020; 4: 176-182.

38 Arabi YM, Fowler R, Hayden FG. Critical care management of adults with community-acquired severe respiratory viral infection. Intensive Care Med 2020; 46: 315-328.

39 Torres A, Loeches IM, Sligl W, et al. Severe flu management: a point of view. Intensive Care Med 2020; 46: $153-162$.

40 Jain S, Kamimoto L, Bramley AM, et al. Hospitalized patients with 2009 H1N1 influenza in the United States, April-June 2009. N Engl J Med 2009; 361: 1935-1944.

41 Katzen J, Kohn R, Houk JL, et al. Early oseltamivir after hospital admission is associated with shortened hospitalization: a five-year analysis of oseltamivir timing and clinical outcomes. Clin Infect Dis 2019; 69: 52-58

42 Díaz E, Rodríguez A, Martin-Loeches I, et al. Impact of obesity in patients infected with 2009 influenza A(H1N1) Chest 2011; 139: 382-386. 